\title{
Differential Expressions of Aquaporin Subtypes in Female Reproductive Tract of Mice
}

\author{
Ji Woo Im ${ }^{1}$, Chae Young Lee ${ }^{1}$, Dong-Hwan Kim², and ${ }^{\dagger}$ Hae-Rahn Bae ${ }^{1,2}$ \\ 'Dept. of Physiology, College of Medicine, Dong-A University, Busan 49201, Korea \\ ${ }^{2}$ Human Life Research Center, Dong-A University, Busan 49315, Korea
}

Received: August 1, 2020

Revised: September 7, 2020

Accepted: September 18, 2020

${ }^{\dagger}$ Corresponding author Hae-Rahn Bae

Dept. of Physiology, Dong-A University College of Medicine, Busan 49201, Korea.

Tel: +82-51-240-2924

Fax: +82-51-245-3872

E-mail:hrbae@dau.ac.kr

Copyright $\odot 2020$ The Korean Society of Developmental Biology.

This is an Open Access article distributed under the terms of the Creative Commons Attribution Non-Commercial License (http://creativecommons.org/licenses/ by-nc/4.0/) which permits unrestricted non-commercial use, distribution, and reproduction in any medium, provided the original work is properly cited.

ORCID

JiWoo Im

https://orcid.org/0000-0002-8053-2669

Chae Young Lee

https://orcid.org/0000-0002-0769-6747

Dong-Hwan Kim

https://orcid.org/0000-0001-6708-645X

Hae-Rahn Bae

https://orcid.org/0000-0002-4261-5666

Conflict of interests

The authors declare no potential conflict of interest.

Acknowledgements

This work was supported by the Dong-A

University research fund.

\section{Authors' contributions}

Conceptualization: Bae HR.

Data curation: Im JW.

Formal analysis: Im JW.

Methodology: Im JW, Lee CY, Kim DW.

Investigation: Bae HR.

Writing - original draft: Im JW, Bae HR.

Writing - review \& editing: Bae HR.

\section{Abstract}

Although many aquaporin (AQP) transcripts have been demonstrated to express in the female reproductive tract, the defined localizations and functions of AQP subtype proteins remain unclear. In this study, we investigated the expression of AQP1, AQP3, AQP5, AQP6, and AQP9 proteins in female reproductive tract of mouse and characterized their precise localizations at the cellular and subcellular levels. Immunofluorescence analyses for AQP1, AQP3, AQP6, and AQP9 showed that these proteins were abundantly expressed in female reproductive tract and that intense immunoreactivities were observed in mucosa epithelial cells with a subtype-specific pattern. The most abundant aquaporin in both vagina and uterine cervix was AQP3. Each of AQP1, AQP3, AQP6, and AQP9 exhibited its distinct distribution in stratified squamous or columnar epithelial cells. AQP9 expression was predominant in oviduct and ovary. AQP1, AQP3, AQP6, and AQP9 proteins were mostly seen in apical membrane of ciliated epithelial cells of the oviduct as well as in both granulosa and theca cells of ovarian follicles. Most of AQP subtypes were also expressed in surface epithelial cells and glandular cells of endometrium in the uterus, but their expression levels were relatively lower than those observed in the vagina, uterine cervix, oviduct and ovary. This is the first study to investigate the expression and localization of 5 AQP subtype proteins simultaneously in female reproductive tract of mouse. Our results suggest that AQP subtypes work together to transport water and glycerol efficiently across the mucosa epithelia for lubrication, proliferation, energy metabolism and $\mathrm{pH}$ regulation in female reproductive tract.

Keywords: Aquaporin 1 (AQP1), AQP3, AQP5, AQP6, AQP9, Female reproductive tract, Immunofluorescence

\section{INTRODUCTION}

Aquaporins (AQPs) are a family of small integral membrane proteins that primarily transport water across the cell membrane along osmotic gradients. Until now, 13 AQPs have been found in mammals (AQP0-12), some of which permit transcellular passage of glycerol and urea as well as water (AQP3, 7, 9, and 10) (Ishibashi et al 2009; Verkman, 2011). AQPs have been demonstrated to play a pivotal role in physiology and pathophysiology of body water balance (Verkman 2005; Verkman, 2012).

Almost all the $\mathrm{AQP}$ transcripts except $\mathrm{AQP0}$ and $\mathrm{AQP} 10$ have been described to express in female 
Ethics approval

The protocols for this study were approved by Dong-A University Medical School Institutional Animal Care Use Committee (DIACUC-07-20). reproductive tract (Huang et al., 2006; Zhu et al., 2015a). AQP0 is expressed exclusively in lens fiber cells of the eye and plays a key player in maintaining proper lens function by regulating water permeability (Hall \& Mathias, 2014), while AQP10 appears to be a pseudogene in some species (Morinaga et al., 2002). In ovary and oviduct, AQP1, AQP2, AQP3, AQP4, AQP5, AQP7, AQP8, AQP9, AQP11, and AQP12 have been reported to express in rodent, but among them AQP1, AQP2, AQP3, AQP4, and AQP8 have been identified in human. AQPs in ovary and oviduct are suggested to play a key role in follicle maturation, blastocyst implantation and ovum transport (Skowronski et al., 2009; Lee et al., 2016). AQP1, AQP3, AQP4, AQP5, AQP7, AQP8, and AQP9 have been detected in uterus and cervix of rodent, but AQP2 instead of AQP5 and $\mathrm{AQP7}$ have been identified in human. AQPs in uterus and cervix are thought to play a crucial role in luminal fluid balance and cervical ripening (Skowronski et al., 2009; Zhu et al., 2015a). AQP1, $\mathrm{AQP} 2, \mathrm{AQP} 3, \mathrm{AQP} 5$, and $\mathrm{AQP} 6$ have been identified in human vagina whereas $\mathrm{AQP} 4, \mathrm{AQP}$, $\mathrm{AQP} 8, \mathrm{AQP} 9, \mathrm{AQP} 11$, and $\mathrm{AQP} 12$ have also been detected in vagina of mouse and rat. AQPs in vagina might be involved in vaginal lubrication (Zhu et al., 2015b).

However, since many studies have been done at the transcript level in female reproductive tract, the presence and localization of AQP subtypes need to be confirmed at the protein level. Moreover, as for some subtypes of AQPs, different results have been reported depending on the species and techniques used (Offenberg et al., 2000; Skowronski et al., 2009; Ma et al., 2016). The comprehensive knowledge regarding the expression pattern of each AQP subtype as well as its cellular and subcellular localization in female reproductive duct can provide much insight to predict its function. In this study, with an aim to elucidate the role of AQPs in female reproduction, we investigated the expression of AQP subtype proteins in female reproductive tract of mouse and characterized their precise localization at the cellular and subcellular levels.

\section{MATERIALSA AND METHODS}

\section{Mice}

All mice were maintained at animal resource center of Dong-A University Medical School under constant temperature and humidity conditions. The protocols for this study were approved by Dong-A University Medical School Institutional Animal Care Use Committee (DIACUC-07-20). Female CD-1 mice aged 30-week-old were used for this study $(n=5)$. Mice were anesthetized by intraperitoneal injection of anesthetized with Avertin (2,2,2-tribromoethanol-tert-amyl alcohol, $250 \mathrm{mg} / \mathrm{kg}$ ). After the uterine horns were exposed by laparotomy, the whole female reproductive organs were meticulously separated. Uterine horns, uterine body, cervix and vagina as well as oviduct were cut transversely, while ovaries were cut horizontally. Tissue samples were embedded in Optimal Cutting Temperature medium (OCT) and rapidly frozen with liquid nitrogen.

\section{Histology}

Unfixed OCT-embedded frozen tissue blocks were sectioned at $5 \mu \mathrm{m}$ thickness with Cryostat (Leica CM1950, Leica Biosystems, Nussloch, Germany). For histological evaluation of each reproductive organ as well as quality verification of the frozen sections, we routinely stained them with hematoxylin and eosin following standard procedures. The sections were mounted, and then observed using microscopy (BX51, Olympus, Tokyo, Japan).

\section{Immunofluorescence}

The frozen sections were air-dried, fixed with ice-cold acetone for $30 \mathrm{~min}$ at $-20^{\circ} \mathrm{C}$ and blocked 
with $1 \%$ bovine serum albumin in PBS containing $0.025 \%$ Tween 20 for $4 \mathrm{~h}$ at room temperature. The sections were incubated with following primary antibodies overnight at $44^{\circ} \mathrm{C}$; anti-AQP1 (1:300, Chemicon, Temecula, CA, USA), anti-AQP3 (1:300, Chemicon), anti-AQP5 (1:100, Alpha Diagnostic Intl., San Antonio, TX, USA), anti-AQP6 (1:300, Chemicon) and anti-AQP9 (1:300, Alpha Diagnostic Intl.) antibodies. Subsequently, the sections were incubated with Alexa Fluor 594-conjugated anti-rabbit IgG H\&L secondary antibody (1:200, Invitrogen, Carlsbad, CA, USA) for $1 \mathrm{~h}$ at room temperature. Finally, the cell nuclei were counterstained with Hoechst 33342 trihydrochloride (1:500, Molecular Probes, Alameda, CA, USA). The sections were mounted with prolong live antifade reagent (Invitrogen) and visualized using laser scanning confocal microscope (LSM 800, Carl Zeiss, Oberkochen, Germany) or fluorescence microscopy (BX51, Olympus).

\section{RESULTS}

\section{Expression and distribution of AQP subtypes in vagina}

We conducted immunofluorescence using antibodies against different AQP subtypes to obtain the detailed information about the localization of AQPs in vagina. Fig. 1 shows that $A Q P 1, A Q P 3$, $\mathrm{AQP} 6$, and $\mathrm{AQP} 9$ were abundantly expressed in vagina, showing a subtype-specific difference in subcellular localization. Anti-AQP1 antibody showed strong labeling in endothelial cells lining blood vessels of different sizes in lamina propria and submucosa (Fig. 1B and H). Moderate intensity of AQP1 labeling was observed in non-keratinized stratified squamous epithelial cells of vaginal mucosa excluding those in the cornified and the basal layers as well as in smooth muscle cells in lamina propria and muscle layers (Fig. 1N). Intensity of AQP3 immunoreactivity was the strongest in mouse vagina among the AQP subtypes we tested (Fig. 1C). Strong AQP3 immunoreactivity was observed in epithelial cells of layers other than the cornified layer (Fig. 1I and O). Anti-AQP3 signal was selectively localized to the plasma membranes of epithelial cells. However, the basement membrane to which the basal layer is attached was not stained by antiAQP3 antibody. Scattered AQP3 labeling was also observed in lamina propria, which is assumed

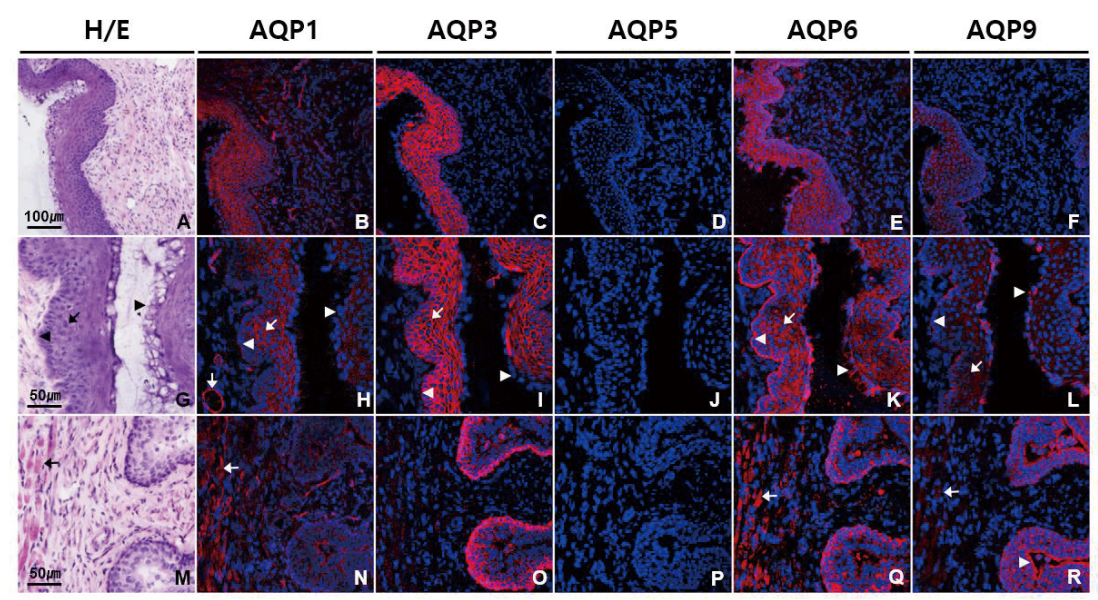

Fig. 1. Immunofluorescence staining of AQP subtypes in frozen sections of the mouse vagina. Hematoxylin and eosin stain $(H / E)$ of the vagina $(A, G, M)$. Immunofluorescence staining for $A Q P 1(B, H, N)$, AQP3 (C, I, O), AQP5 (D, J, P), AQP6 (E, K, Q) and AQP9 (F, L, R) in the vagina. Down-left arrows indicate epithelial cells in spinous layers, while rightwards and leftwards arrowheads indicate epithelial cells in cornified layers and the basement membrane, respectively. Leftwards arrows indicate smooth muscle cells, while a downwards arrow indicates endothelial cells. AQP, aquaporin. 
to be associated with inflammatory cells. No significant AQP5 immunoreactivity was detected in mouse vagina (Fig. 1D, J, and P). AQP6 labeling was prominent throughout all the epithelial cells including those in basal and cornified layers (Fig. 1E and K). AQP6 immunoreactivity was distributed in the cytoplasm of epithelial cells, not the cell membrane. But interestingly, anti-AQP6 antibody uniquely stained the basement membrane, demarcating the border between vaginal epithelia and the underlying connective tissue. AQP6 immunoreactivity was also found in stroma and smooth muscle cells (Fig. 1Q). Anti-AQP9 antibody stained not only the cytoplasms of all the epithelial cells including cornified layers but also basement membrane, as did anti-AQP6 antibody (Fig. 1F, L, and R). However, AQP9 immunoreactivity was more intense in cornified layers than basement membrane, while AQP6 immunoreactivity was more pronounced in basement membrane than cornified layers. Weak AQP9 labeling was also observed in muscle layers.

\section{Expression and distribution of $A Q P$ subtypes in uterus and cervix}

In ectocervix of uterus, AQP1, AQP3, AQP6, and AQP9 were highly expressed especially in stratified squamous epithelial cells of mucosa, and the expression patterns of $\mathrm{AQP}$ subtypes were similar to those in vagina (Fig. 2B-F). In endocervix, all the AQPs tested were differentially expressed in mucosa. Weak positive AQP1 immunoreactivity was found in endocervical columnar epithelia as well as underlying blood vessels (Fig. $2 \mathrm{H}, \mathrm{N}$, and T). AQP3 immunoreactivity was the strongest among the AQPs tested in endocervical epithelia, and specific to basolateral and basal surface of both columnar epithelial and basal cells (Fig. 2C, I, O, and U). Weak but clear anti-AQP5 labeling was observed at the basement membrane of endocervical epithelia (Fig. 2D, $\mathrm{J}, \mathrm{P}$, and V). AQP6 immunostaining was noted in all layers of endocervix, but more abundant in endocervical epithelia. AQP6 immunostaining in epithelia was widely dispersed, extending from

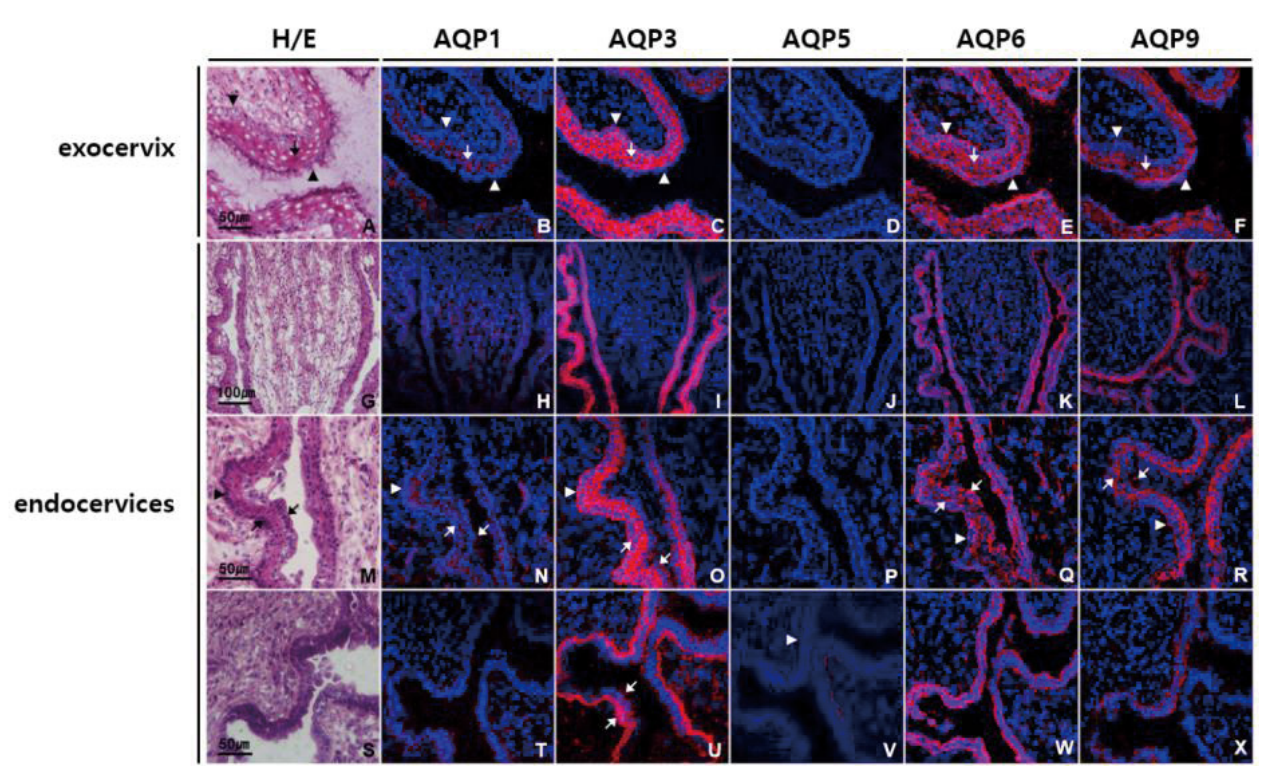

Fig. 2. Immunofluorescence staining of AQP subtypes in frozen sections of the mouse uterine cervix. Hematoxylin and eosin stain $(H / E)$ of the exocervix $(A)$ and the endocervix $(G, M, S)$. Immunofluorescence staining for AQP1 (B), AQP3 (C), AQP5 (D), AQP6 (E) and AQP9 (F) in the exocervix. Immunofluorescence staining for AQP1 $(\mathrm{H}, \mathrm{N}, \mathrm{T})$, AQP3 $(\mathrm{I}, \mathrm{O}, \mathrm{U}), \mathrm{AQP5}(\mathrm{J}, \mathrm{P}, \mathrm{V}), \mathrm{AQP} 6(\mathrm{~K}, \mathrm{Q}$, $W)$ and $A Q P 9(L, R, X)$ in the endocervix. Downwards arrows indicate epithelial cells in spinous layers, while upwards and downwards arrowheads indicate epithelial cells in cornified layers and basement membrane, respectively (A-F). Down-left and up-right arrows indicate columnar epithelial and basal cells, respectively, while the right arrowheads, indicate the basement membrane $(M-X)$. AQP, aquaporin. 
the base to the apical surface (Fig. $2 \mathrm{E}, \mathrm{K}, \mathrm{Q}$, and $\mathrm{W}$ ). In contrast, anti-AQP9 labeling was more intensely distributed at the apical region of columnar epithelia than the basal cell layer (Fig. 2F, L, R, and X).

In uterine horn, subtype-specific difference in $\mathrm{AQP}$ expression was similar to that of endocervix (Fig. 3). However, the intensity of AQP immunostaining in uterine horn was low in general compared to that in endocervix. Immunoreactivities of AQP1, AQP3, AQP6, and AQP9 protein were prominent in endometrium, especially at surface epithelial cells and glandular cells. AntiAQP3 and anti-AQP9 signals were stronger than the others and more condensed at the apical membranes than basolateral membranes of endometrial glandular cells. AQP5 immunoreactivity was detected only a certain type of cells in endometrial glands (Fig. 3D,J, and P).

\section{Expression and distribution of $A Q P$ subtypes in oviduct and ovary}

In oviduct, $\mathrm{AQP} 1, \mathrm{AQP} 3, \mathrm{AQP} 6$, and $\mathrm{AQP} 9$ were strongly expressed especially in ciliated columnar epithelial cells (Fig. 4). Immunoreactivities of AQP1, AQP3, AQP6, and AQP9 were localized mainly to cilia and luminal surface of epithelial cells with a subtle subtype-specific difference. Both anti-AQP3 and anti-AQP6 signals were confined to the apical side of oviduct epithelial cells. However, anti-AQP1 signal was also observed the cytoplasm and basement membrane of epithelial cells, blood vessels in lamina propria and submucosa as well as smooth muscles (Fig. 4B, H, and N). Anti-AQP9 signal was the strongest among the AQPs tested in oviduct epithelial cells, but also detected in basolateral and basement membrane of epithelial cells as well as the mesothelial cells of the oviduct serosa (Fig. 4F, L, and R). However, AQP5 immunoreactivity was not identified in the mouse oviduct (Fig. 4D, J, and P).

In ovary, moderate intensity of $\mathrm{AQP} 1, \mathrm{AQP} 3, \mathrm{AQP} 6$, and $\mathrm{AQP} 9$ labeling was observed in ovarian follicles of different stages and sizes (Fig. 5). AQP1, AQP3, AQP6, and AQP9 proteins were equally expressed in all the types of cells in preantral follicles, that is, an oocyte, granular cells and theca cells (Fig. 5B-F). In antral follicles, anti-AQP1 and anti-AQP6 signals were of similar strength in both granular and theca cells, while anti-AQP3 and anti-AQP9 signals were stronger in theca cells than granular cells (Fig. $5 \mathrm{H}-\mathrm{L}$ and N-R). Corpus luteum was the one in which AQP1,

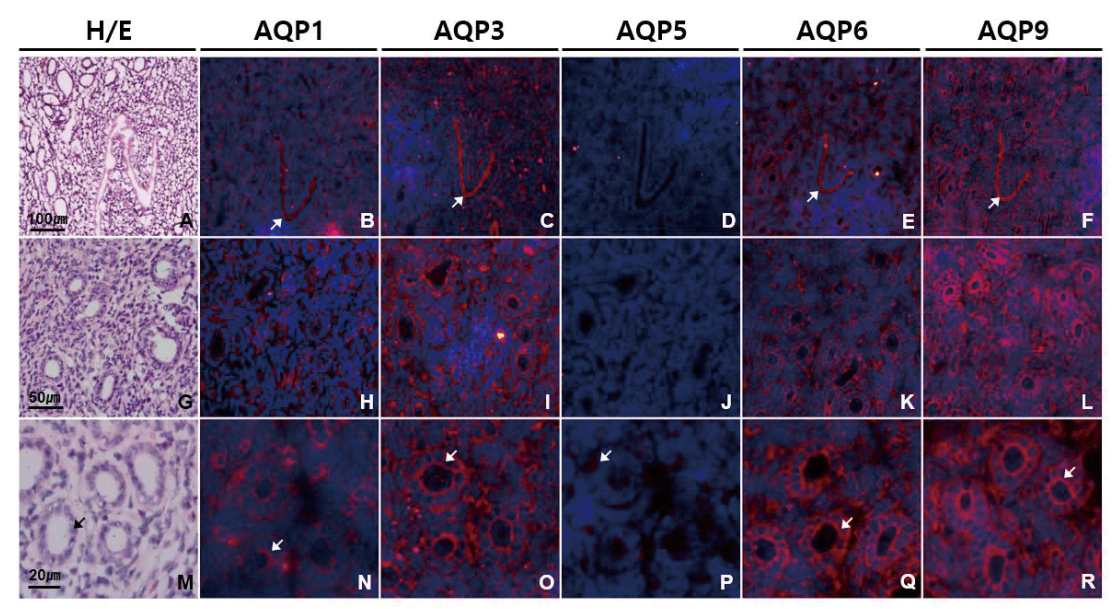

Fig. 3. Immunofluorescence staining of AQP subtypes in frozen sections of the mouse uterus horn. Hematoxylin and eosin stain (H/E) of the uterus horn (A, G, M). Immunofluorescence staining for AQP1 $(B, H, N), A Q P 3(C, I, O), A Q P 5(D, J, P), A Q P 6(E, K, Q)$ and AQP9 $(E, L, R)$ in the uterus horn. Up-right and down-left arrows indicate apical membranes of surface epithelial and glandular cells of endometrium, respectively. AQP, aquaporin. 


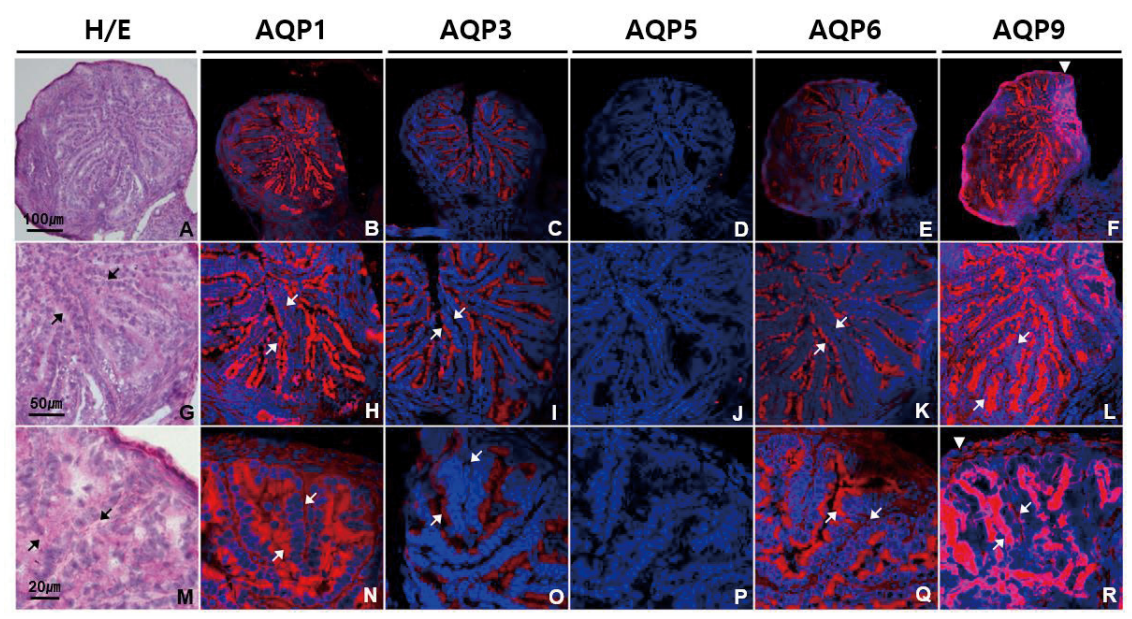

Fig. 4. Immunofluorescence staining of AQP subtypes in frozen sections of the mouse oviduct. Hematoxylin and eosin stain $(H / E)$ of the oviduct $(A, G, M)$. Immunofluorescence staining for $A Q P 1(B, H, N)$, AQP3 (C, I, O), AQP5 (D, J, P), AQP6 (E, K, Q) and AQP9 (E, L, R) in the oviduct. Up-right and down-left arrows indicate cilia of columnar epithelial cells and lamina propria, respectively. Downwards arrowheads indicate the mesothelial cells of the serosa. AQP, aquaporin.

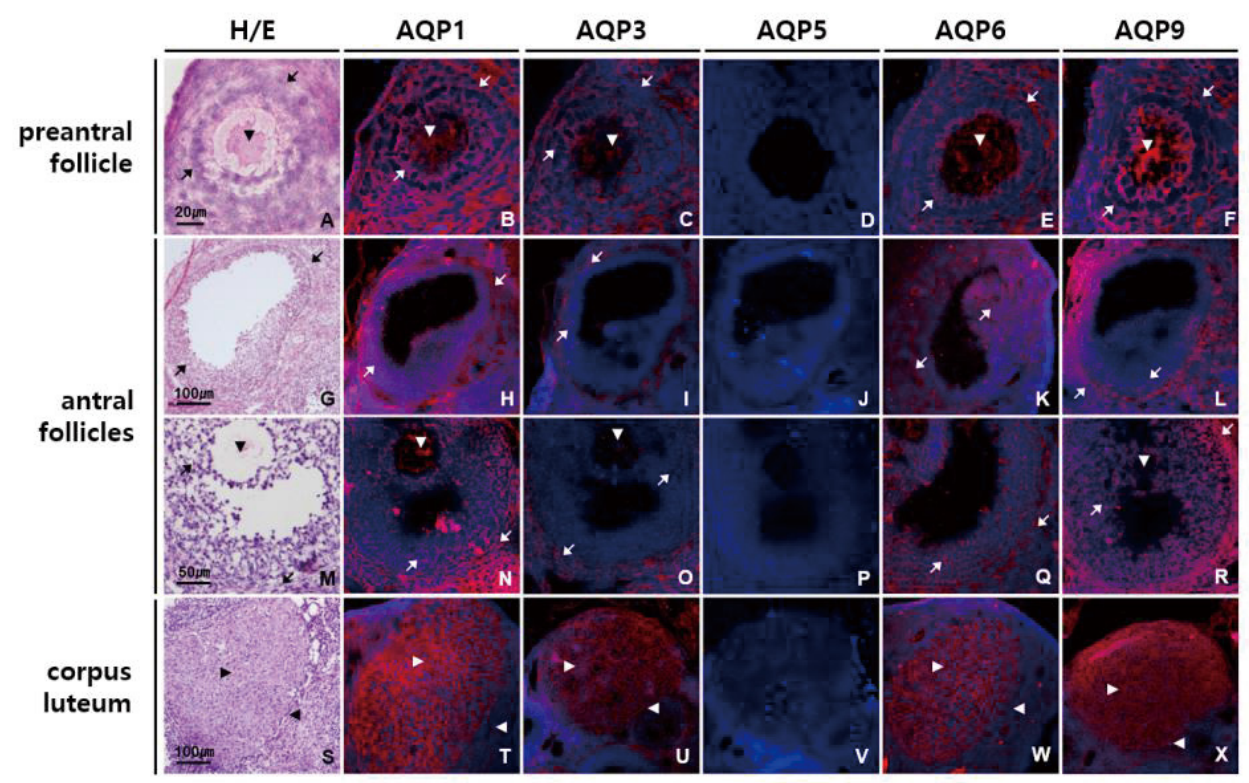

Fig. 5. Immunofluorescence staining of AQP subtypes in frozen sections of the mouse ovarian follicles. Hematoxylin and eosin stain (H/E) of ovarian follicles at different stages; preantral follicle $(A)$, antral follicle $(G, M)$ and corpus luteum (S). Immunofluorescence staining for AQP1 $(B, H, N, T), A Q P 3(C, I, O, U)$, AQP5 (D, J, P, V), AQP6 (E, K, Q, W) and AQP9 (E, L, R, X). Up-right and down-left arrows indicate granulosa and theca cells, respectively. Downwards arrowheads indicate oocytes. Rightwards and leftwards arrowheads indicate granulosa lutein and theca lutein cells, respectively. AQP, aquaporin.

AQP3, AQP6, and AQP9 proteins were most abundantly expressed (Fig. 5T-X). Although both granulosa lutein and theca lutein cells expressed them, immunoreactivities to these AQP subtypes were more pronounced in granulosa lutein cells than theca lutein cells. AQP5 immunoreactivity was not detected in the mouse ovary. 


\section{DISCUSSION}

We conducted this study to gain detailed knowledge of the presence and localization of AQP subtypes in female reproductive duct. Immunofluorescence analysis revealed that AQP1, AQP3, AQP6, and AQP9 proteins were abundantly expressed in mouse female reproductive tract and these AQP subtypes were strongly expressed especially in mucosa epithelial cells with subtypespecific localization pattern depending on the parts of the tract. In both vagina and uterine cervix, AQP3 was the most expressed among AQPs tested and each of AQP1, AQP3, AQP6, and AQP9 exhibited its distinct distribution in stratified squamous and columnar epithelial cells, respectively. In oviduct and ovary, AQP9 was the most expressed and all of AQP1, AQP3, AQP6, and AQP9 proteins were concentrated in apical membrane of ciliated oviduct epithelial cells and in both granulosa and theca cells of ovarian follicles. Uterus also expressed these AQP subtypes in surface epithelial cells and glandular cells, but their expression levels are relatively lower than those of the vagina, cervix, oviduct and ovary.

AQP3 is a membrane transporting protein facilitating water and glycerol transport. AQP3 is highly expressed in the basolateral membranes of epithelial cells in kidney collecting duct, airways, intestine and epidermis (Verkman, 2005). Mice lacking AQP3 manifest various degrees of nephrogenic diabetes insipidus resulting from inability to concentrate the urine, and dry skin with decreased elasticity and impaired biosynthesis due to reduced glycerol and water content in epidermis (Ma et al., 2000; Hara et al., 2002). In a previous paper, we reported that AQP3 expresses in the visceral yolk sac cells of fetal membrane, the syncytiotrophoblasts of the labyrinthine placenta and fetal nucleated red blood cell membrane and that AQP3 null mice exhibits reduced intrauterine fetal growth (Seo et al., 2018). This study shows that AQP3 is highly expressed in apical surface of oviduct epithelial cells in mouse, which is the first reported finding. Yang et al. (2016) reported AQP3 expression in chicken oviduct and its regulation by estrogen and Nah et al. (2015) detected AQP3 mRNA in mouse oviduct. However, we have confirmed the presence of AQP3 proteins in mouse oviduct mucosa and characterized their cellular and subcellular localization in this study. It has been reported that AQP1 and AQP3 promote cell proliferation (Galán-Cobo et al., 2015 \& 2016) and that AQP1, AQP3 and AQP9 are involved in cancer progression (Dajani et al., 2018). Therefore, it can be speculated that $\mathrm{AQP} 1, \mathrm{AQP} 3$, and $\mathrm{AQP} 9$ present in ovarian follicles and corpus luteum contribute the rapid growth of follicles during folliculogenesis as well as follicular fluid accumulation and nutrient supply to granulosa cells and oocytes.

AQP6 was originally identified as a homolog of AQP2 and expresses mainly in the intracellular vesicles of acid-secreting type A-intercalated cells of renal collecting ducts, colocalizing with $\mathrm{H}^{+}$ATPase (Ohshiro et al., 2001). AQP6 is permeable to anionic ions, especially nitrate, as well as water (Ikeda et al., 2002). Co-localization of AQP6 with a V-type $\mathrm{H}^{+}$-ATPase suggests a role in intracellular vesicle acidification and acid secretion into lumen (Yasui, 2009). In female reproductive tract, expression of AQP6 protein has not been reported except human vagina (Kim et al., 2011). In this study we first demonstrated that AQP6 protein is abundantly expressed throughout female reproductive tract including vagina. $\mathrm{AQP} 6$ might play a role in maintaining $\mathrm{pH}$ homeostasis in female reproductive tract.

AQP9 is a member of the aquaglyceroporin family and shares high homology with AQP3 (Rojek et al., 2007). In addition to water, AQP9 transports not only small uncharged molecules like glycerol, urea, purines, and pyrimidines, but also multiple neutral and ionic arsenic species including arsenic trioxide, monomethylarsenous acid and dimethylarsenic acid (Geng et al., 2017). AQP9 expresses in liver, leukocytes, brain and testis and is important for hepatic glycerol metabolism (Calamita et al., 2018). We ascertain here for the first time that AQP9 protein is prominently 
expressed in mouse vagina and cervix, peculiarly at the luminal surface of epithelial cells. The unique location of AQP9 in mouse vagina and cervix along with that of AQP3 imply that these 2 aquaglyceroporins work in concert to transport water and glycerol efficiently across the mucosa epithelia for energy metabolism as well as hydration and barrier function in vaginal and cervix. Both AQP3 and AQP9 might cooperate together in this way in oviduct epithelium, ovarian follicles and corpus luteum where they are highly co-expressed.

Taken together, this is the first study investigating simultaneously the expression of $5 \mathrm{AQP}$ subtype proteins in mouse female reproductive tract. We found that AQP1, AQP3, AQP6, and AQP9 proteins are abundantly expressed in mouse female reproductive tract and that these AQP subtypes are strongly expressed especially in mucosa epithelial cells with subtype-specific localization pattern. Our results suggest that several AQP subtypes work in concert to transport water and glycerol efficiently across the mucosa epithelia for lubrication, proliferation, energy metabolism and $\mathrm{pH}$ regulation in female reproductive tract. Further study on the expressional change of $\mathrm{AQP}$ subtypes during different stages of estrous cycle is required to get comprehensive knowledge about the function of $\mathrm{AQP}$ subtypes in female reproductive tract.

\section{REFERENCES}

Calamita G, Perret J, Delporte C (2018) Aquaglyceroporins: Drug targets for metabolic diseases? Front Physiol 9:851.

Dajani S, Saripalli A, Sharma-Walia N (2018) Water transport proteins-aquaporins (AQPs) in cancer biology. Oncotarget 9:36392-36405.

Galán-Cobo A, Ramírez-Lorca R, Serna A, Echevarría M (2015) Overexpression of AQP3 modifies the cell cycle and the proliferation rate of mammalian cells in culture. PLoS One 10:e0137692.

Galán-Cobo A, Ramírez-Lorca R, Toledo-Aral JJ, Echevarría M (2016) Aquaporin-1 plays important role in proliferation by affecting cell cycle progression. J Cell Physiol 231:243-256.

Geng X, McDermott J, Lundgren J, Liu L, Tsai KJ, Shen J, Liu Z (2017) Role of AQP9 in transport of monomethyselenic acid and selenite. Biometals 30:747-755.

Hall JE, Mathias RT (2014) The aquaporin zero puzzle. Biophys J 107:10-15.

Hara M, Ma T, Verkman AS (2002) Selectively reduced glycerol in skin of aquaporin-3-deficienct mice may account for impaired skin hydration, elasticity, and barrier recovery. J Biol Chem 227:46616-46621.

Huang HF, He RH, Sun CC, Zhang Y, Meng QX, Ma YY (2006) Function of aquaporins in female and male reproductive systems. Hum Reprod Update 12:785-795.

Ikeda M, Beitz E, Kozono D, Guggino WB, Agre P, Yasui M (2002) Characterization of aquaporin-6 as a nitrate channel in mammalian cells. Requirement of pore-lining residue threonine 63.J Biol Chem 277:39873-39879.

Ishibashi K, Hara S, Kondo S (2009) Aquaporin water channels in mammals. Clin Exp Nephol 13:107-117.

Kim SO, Oh KJ, Lee HS, Ahn K, Kim SW, Park K (2011) Expression of aquaporin water channels in the vagina in premenopausal women.J Sex Med 8:1925-1930.

Lee HJ, Jee BC, Kim SK, Kim H, Lee JR, Suh CS, Kim SH (2016) Expressions of aquaporin family in human luteinized granulosa cells and their correlations with IVF outcomes. Hum Reprod 31:822-831.

Ma J, Zhou C, Yang J, Ding X, Zhu Y, Chen X (2016) Expression of AQP6 and AQP8 in 
epithelial ovarian tumor.J Mol Histol 47:129-134

Ma T, Song Y, Yang B, Gillespie A, Carlson EJ, Epstein CJ, Verkman AS (2000) Nephrogenic diabetes insipidus in mice lacking aquaporin-3 water channels. Proc Natl Acad Sci USA 97:4386-4391.

Morinaga T, Nakakoshi M, Hirao A, Imai M, Ishibashi K (2002) Mouse aquaporin 10 gene (AQP10) is a pseudogene. Biochem Biophys Res Commun 294:630-634.

Nah WH, Oh YS, Hwang JH, Gye MC (2015) Changes in aquaporin 5 in the non-ciliated cells of mouse oviduct according to sexual maturation and oestrous cycle. Reprod Fertil Dev 29:336344.

Offenberg H, Barcroft LC, Caveney A, Viuff D, Thomsen PD, Watson AJ (2000) mRNAs encoding aquaporins are present during murine preimplantation development. Mol Reprod Dev 57:323-330.

Ohshiro K, Yaoita E, Yoshida Y, Fujinaka H, Matsuki A, Kamiie J, Kovalenko P, Yamamoto T (2001) Expression and immunolocalization of AQP6 in intercalated cells of the rat kidney collecting duct. Arch Histol Cytol 64:329-338.

Rojek AM, Skowronski MT, Füchtbauer EM, Füchtbauer AC, Fenton RA, Agre P, Frøkiaer J, Nielsen S (2007) Defective glycerol metabolism in aquaporin 9 (AQP9) knockout mice. Proc Natl Acad Sci USA 104:3609-3614.

Seo MJ, Lim JH, Kim DH, Bae HR (2018) Loss of aquaporin-3 in placenta and fetal membranes induces growth restriction in mice. Dev Reprod 22:263-273.

Skowronski MT, Kwon TH, Nielsen S (2009) Immunolocalization of aquaporin 1, 5, and 9 in the female pig reproductive system.J Histochem Cytochem 57:61-67.

Verkman AS (2005) Novel roles of aquaporins revealed by phenotype analysis of knock-out mice. Rev Physiol Biochem Pharmacol 155:31-55.

Verkman AS (2011) Aquaporins at a glance.J Cell Sci 124:2107-2112.

Verkman AS (2012) Aquaporins in clinical medicine. Annu Rev Med 63:303-316.

Yang C, Lim W, Bae H, Song G (2016) Aquaporin 3 is regulated by estrogen in the chicken oviduct and is involved in progression of epithelial cell-derived ovarian carcinomas. Domest Anim Endocrinol 55:97-106.

Yasui M (2009) pH regulated anion permeability of aquaporin-6. Handb Exp Pharmacol 190:299308.

Zhu C, Jiang Z, Bazer FW, Johnson GA, Burghardt RC, Wu G (2015a) Aquaporins in the female reproductive system of mammals. Front Biosci (Landmark Ed) 20:838-871.

Zhu J, Xia J, Jiang J, Jiang R, He Y, Lin H (2015b) Effects of estrogen deprivation on expression of aquaporins in rat vagina. Menopause 22:893-898. 
